\title{
GENETIC COMPUTATION OF GEODESICS ON THREE-DIMENSIONAL CURVED SURFACES
}

\author{
Professor B Porter, Dr S S Mohamed, \\ and Professor T R Crossley
}

\author{
University of Salford, UK
}

\begin{abstract}
Most classical approaches to the determination of geodesics (such as the calculus of variations) are difficult to apply except for simple surfaces. Genetic algorithms are therefore used to provide a general methodology for the computation of geodesics.
\end{abstract}

\section{INTRODUCTION}

Many important problems in engineering involve the computation of geodesics (ie, lines of shortest distance) on three-dimensional curved surfaces. Thus, for example, it may be required to position a robotically operated riveting machine so as to produce a set of equally spaced rivets along the geodesic joining two points on the curved fuselage of an aircraft under manufacture. However, most classical approaches to the determination of geodesics (such as the calculus of variations) are difficult to apply except for simple surfaces. In addition, the outputs from such procedures are usually in the form of mathematical functions and not in the form of discrete sets of Cartesian co-ordinates suitable for numerical control (or, more generally, for use in digitised form within any CAD/CAM system).

The need to generate geodesics in such digitised forms has been addressed by, for example, Kiryati and Szekely [1]. However, it is considered that such procedures are relatively difficult to apply (particularly in the case of complicated curved surfaces). In this paper, genetic algorithms [2][3] are therefore used to provide a general methodology for the computation of geodesics. The outputs of the sets of Cartesian co-ordinates from this genetic procedure are in a form immediately suited to such practical tasks as the programming of $\mathrm{CNC}$ machine tools or industrial robots. The effectiveness of this genetic methodology is illustrated by reference to a spherical surface, for which theoretical results are known.

\section{GENETIC METHODOLOGY}

The general methodology for the genetic computation of geodesics can be conveniently described in relation to a curved surface, $\Xi$, described by the equation

$$
f(x, y, z)=0
$$

in three-dimensional Cartesian space. In equation (1), $P \equiv(x, y, z)$ is a point referred to fixed rectangular $O x, O y, O z$ axes with $O$ as origin. It is desired to find the line of shortest length joining the points

$$
A \equiv\left(x_{A}, y_{A}, z_{A}\right)
$$

and

$$
B \equiv\left(x_{B}, y_{B}, z_{B}\right)
$$

which lies on the surface described by equation (1). Since the points $A$ and $B$ lie on this surface, it is evident that

$$
f\left(x_{A}, y_{A}, z_{A}\right)=0
$$

and 


$$
f\left(x_{B}, y_{B}, z_{B}\right)=0 \text {. }
$$

It is convenient to define the geodesic joining $A$ and $B$ on $\Xi$ in terms of $n$ equally spaced interior points

$P_{i} \equiv\left(x_{i}, y_{i}, z_{i}\right) \quad(i=1,2, \ldots, n) \ldots \ldots(s)$

Thus, if

$$
\begin{aligned}
& \qquad l_{1}=d\left(A, P_{1}\right), \quad \ldots(\delta a) \\
& l_{i}=d\left(P_{i-1}, P_{i}\right) \quad(i=2,3, \ldots, n), \ldots(\delta b) \\
& \text { and }
\end{aligned}
$$

$$
l_{n+1}=d\left(P_{n}, B\right)
$$

are the lengths of rectilinear segments between $A$ and $P_{1}, P_{1}$ and $P_{2}, \ldots$, and $P_{n}$ and $B$, respectively, these interior points are required to be such that

$$
l=\sum_{i=1}^{n+1} l_{i}
$$

is a minimum where

$$
l_{1}=l_{2}=\ldots=l_{n+1}
$$

and

$f\left(x_{i}, y_{i}, z_{i}\right)=0 \quad(i=1,2, \ldots, n) \ldots \ldots(9)$

This constrained minimisation problem is equivalent to determining the Cartesian co-ordinates of the $n$ interior points $P_{1}, P_{2}, \ldots, P_{n}$ such that the cost function

$$
\begin{aligned}
\Gamma= & \mu_{1} l+\mu_{2} \sum_{i=1}^{n+1}\left|\left(l_{i}-\bar{l}\right)\right| \\
& +\mu_{3} \sum_{i=1}^{n}\left|f\left(x_{i}, y_{i} z_{i}\right)\right|
\end{aligned}
$$

is minimised. In equation (10),

$$
\bar{l}=\frac{l}{n+1} \quad, \quad \ldots \text { (11) }
$$

and $\mu_{1}, \mu_{2}$, and $\mu_{3}$ are positive weighting parameters. It is evident that, in the case of curved surfaces for which geodesics are uniquely defined, the Cartesian co-ordinates of the $n$ interior points that minimise $\Gamma$ will produce a space-polygonal approximation to the required geodesic in which the sides of the space polygon are of equal length and its vertices lie on $\Xi$. In order to use genetic algorithms to determine this geodesic, it is necessary only to encode the Cartesian co-ordinates of the $n$ triples $\left(x_{1}, y_{1}, z_{1}\right)$, $\left(x_{2}, y_{2}, z_{2}\right), \ldots,\left(x_{n}, y_{n}, z_{n}\right)$ in accordance with a system of concatenated, multi-parameter, mapped, fixed-point coding [3]. Thus, each ordered set of $n$ such triples is represented by a string of binary digits with $3 n$ sub-strings. Then, following any choice of an initial generation of such strings, successive generations of strings can be readily obtained using the basic genetic operations of selection, crossover, and mutation [3]. In particular, by defining, the 'fitness' of each such string as

$$
\Phi=1 / \Gamma \quad,
$$

the successive generations of interior points $P_{1}, P_{2}, \ldots, P_{n}$ produced by the genetic algorithm tend to exhibit progressively increasing 'fitness', $\Phi$, as the cost function, $\Gamma$, decreases towards its minimum value. This methodology for the genetic computation of geodesics thus produces space-polygonal approximations to the required geodesics that satisfy the constraints expressed by equations (8) and (9) with increasing accuracy while minimising the length defined by equation (7). Note that, although the constraint expressed by equation (8) is appropriate to many manufacturing applications of geodesics (in riveting, for example), this particular constraint is not fundamental to the general methodology. Note also that planar problems, such as those involved in designing shop-floor layouts or in optimising the paths of remotely guided vehicles, can be solved according to this formalisation simply by replacing equation (9) with the constraints

$$
z_{i}=0 \quad(i=1,2, \ldots, n) . \quad \ldots\left(9^{\prime}\right)
$$

\section{ILLUSTRATIVE EXAMPLE}

This general methodology for the genetic computation of geodesics can be conveniently illustrated by reference to a 
curved surface, $\Xi$, for which theoretical results are known. Thus, for example, consider the case of a sphere with centre at the origin and of unit radius for which it is required to determine the geodesic joining the points

$$
A \equiv(1,0,0) \quad \ldots(13 a)
$$

and

$$
B \equiv(0,1,0) \quad . \quad \ldots(13 b)
$$

In this case, let it be desired to determine the three interior points

$$
\begin{array}{ll}
P_{1} \equiv\left(x_{1}, y_{1}, z_{1}\right), & \ldots(14 a) \\
P_{2} \equiv\left(x_{2}, y_{2}, z_{2}\right), & \ldots(14 b)
\end{array}
$$

and

$$
P_{3} \equiv\left(x_{3}, y_{3}, z_{3}\right) \quad \ldots(14 c)
$$

which satisfy the constraints expressed by equations (8) and (9), and which minimise the length defined by equation (7), in case $n=3$. The results of the genetic computation in this case are shown in Figures 1 and 2 over 100,000 generations for a population size $N=100$, a crossover probability $p_{c}=0.6$, a mutation probability $p_{m}=0.001$, and a string length $\lambda=90$. In Figure 1 , the best-of-generation and generation-average values of the cost function, $\Gamma$, are plotted against generation number when $\mu_{1}=2.5, \mu_{2}=1$, and $\mu_{3}=1$; whilst, in Figure 2 , the associated best-of-generation values of the nine Cartesian co-ordinates $x_{1}, y_{1}, z_{1}, x_{2}, y_{2}, z_{2}, x_{3}, y_{3}, z_{3}$ are plotted against generation number. It is thus evident that the optimal locations of the three interior points are

$$
\begin{array}{r}
P_{1} \equiv(0.92375,0.38221,-0.00098), \\
\ldots(15 a) \\
P_{2} \equiv(0.70869,0.70478,0.00098),
\end{array}
$$

and

$$
P_{3} \equiv(0.38416,0.92179,0.00098)
$$

for which

$$
\begin{aligned}
& l=1.55989 \quad, \quad \ldots(16) \\
& l_{1}=0.38974 \quad, \quad \ldots(17 a) \\
& l_{2}=0.38769 \quad, \quad \ldots(17 b) \\
& l_{3}=0.39040 \quad, \quad \ldots(17 c)
\end{aligned}
$$

and

$$
l_{4}=0.39204 \quad . \quad \ldots(17 d)
$$

These genetically computed results are evidently close to the corresponding theoretical values

$$
\begin{aligned}
& P_{1} \equiv(0.92387,0.38268,0) \\
& \ldots(18 a) \\
& P_{2} \equiv(0.70710,0.70710,0) \\
& \ldots(l 8 b) \\
& P_{3} \equiv(0.38268,0.92387,0) \quad, \quad \ldots(18 c) \\
& l_{l}=1.56071 \quad, \quad \ldots(19) \\
& l_{1}=0.39017 \quad, \quad \ldots(20 a) \\
& l_{2}=0.39017 \quad, \quad \ldots(20 b) \\
& l_{3}=0.39017 \quad, \quad \ldots(20 c)
\end{aligned}
$$

and

$$
l_{4}=0.39017
$$

This accuracy can be improved, if desired, by using longer strings. Note that the outputs of the sets of Cartesian co-ordinates from this genetic procedure are in a form immediately suited to the programming of $\mathrm{CNC}$ machine tools or industrial robots. In addition, note that 
the evolutionary process depicted in Figures 1 and 2 can be accelerated by using more precise a priori information regarding the admissible ranges of the Cartesian co-ordinates $x_{i}, y_{i}, z_{i}(i=1,2, \ldots, 3)$.

\section{CONCLUSION}

In this paper, genetic algorithms have been used to provide a general methodology for the computation of geodesics on three-dimensional curved surfaces. The effectiveness of this genetic methodology has been illustrated by reference to a spherical surface for which theoretical results are known.

\section{REFERENCES}

[1] N Kiryati and G Szekely, "Estimating shortest paths and minimal distances on a digitised three-dimensional surface", Pattern Recognition, Vol 26, pp 1623-1637, 1993.

[2] J H Holland, "Adaptation in Natural and Artificial Systems", University of Michigan Press, 1975.

[3] D E Goldberg, "Genetic Algorithms in Search, Optimization, and Machine Learning", Addison-Wesley, 1989. 

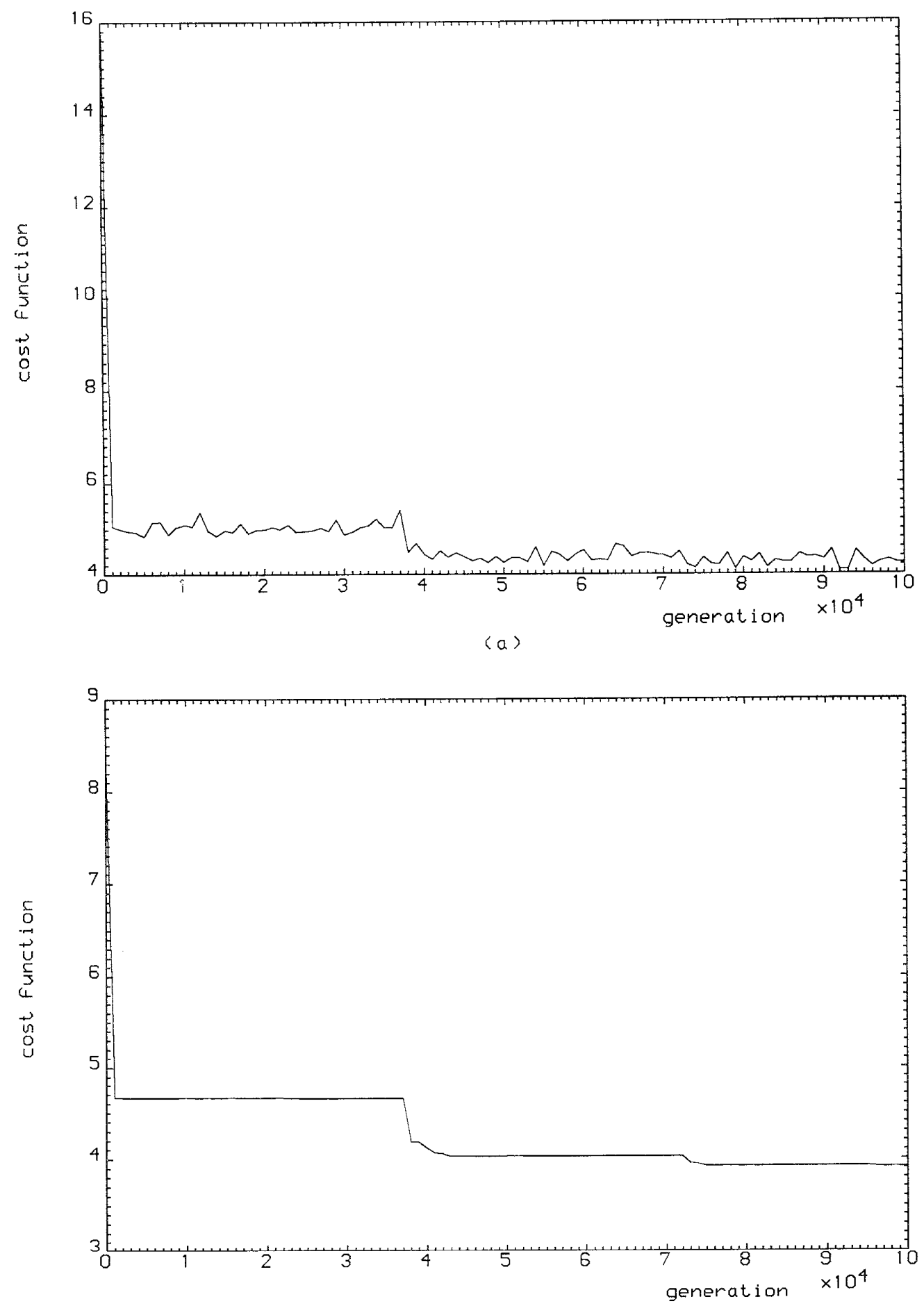

(b)

Fig. $1:(a)$ Generation-average cost Function. (b) Best-of-generation cost function. 

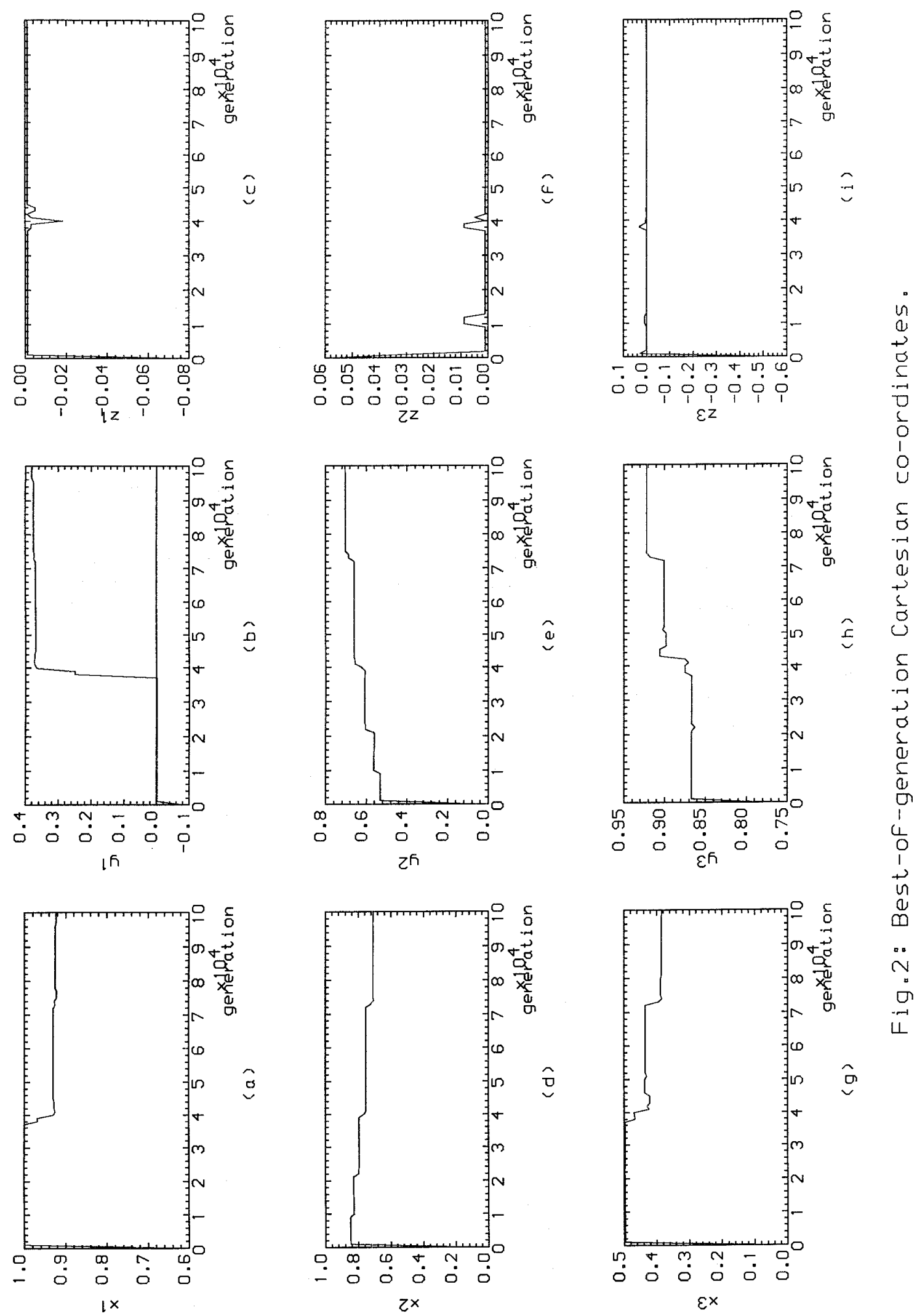